\title{
Editorial for Special Issue
}

\section{Uwe T. Bornscheuer* \\ (Chemo-) enzymatic cascade reactions}

\author{
https://doi.org/10.1515/znc-2019-0016
}

The past decade has seen a substantial increase in successful examples for the combination of biocatalytic reaction steps in cascade reactions, preferentially in one-pot for multistep syntheses [1, 2]. Furthermore, many published examples also dealt with chemoenzymatic reactions where transition metal-, photo-, and organo-catalysis were successfully combined with enzymatic steps [3]. Major driving forces have been progress in enzyme discovery and engineering, but also the development of conditions compatible with different types of (bio)catalysts as well as innovative reactor types. Important outcomes of cascade reactions are higher yields, decreased costs, and environmental benefits matched with high selectivity. These obvious advantages make the concept of cascade reactions highly attractive for the pharmaceutical, chemical, and flavor industry.

This motivated us to publish this dedicated issue about (chemo-) enzymatic cascades in the Zeitschrift für Naturforschung. We were happy to have a total of six contributions, covering different types of enzymes to synthesise a variety of target compounds useful for, e.g. human nutrition, as flavors or fragrances or even polymers and their precursors, as well as reaction engineering concepts.

Peters and Buller combined in their contribution an ene reductase with a squalene hopene cyclase and could demonstrate that the conversion of citral to (-)-iso-isopulegol is feasible by this cascade reaction [4]. In our own contribution, we have studied a 3-enzyme cascade reaction to make 6-hydroxyhexanoic acid from cyclohexanol. An alcohol dehydrogenase and a Baeyer-Villiger monooxygenase produced recombinantly in Escherichia coli catalyze the first two steps to afford $\varepsilon$-caprolactone, which is then hydrolysed by lipase CAL-B to the target product thus overcoming product inhibition resulting in high productivities of the overall process [5].

The group of Woodley presents concepts for the design of enzyme cascade processes for the production

\footnotetext{
*Corresponding author: Uwe T. Bornscheuer, Department of Biotechnology and Enzyme Catalysis, Institute of Biochemistry, University of Greifswald, Felix-Hausdorff-Str. 4, Greifswald 17487 , Germany, Phone: +49 3834420 4367,

E-mail: uwe.bornscheuer@uni-greifswald.de
}

of low-priced chemicals [6]. They suggest that groups of enzymes should be combined in modules, which then facilitate downstream processing and gives higher overall yields as exemplified for gluconic acid.

In the rapid communication by Muschiol and Meyer they exemplify a chemoenzymatic approach in which the human milk oligosaccharide (HMO) precursor lacto- $\mathrm{N}$ triose II and three regioisomers could be synthesized in a sequential cascade using $\beta$ - $\mathrm{N}$-acetylhexosaminidases from the glycoside hydrolase GH20 family [7].

Syrén and coworkers used the readily available monoterpene Norcamphor as a model compound, which is converted in a chemoenzymatic reaction using a Baeyer-Villiger monooxygenase-catalysed transformation as key steps to afford the corresponding lactone and its polyesters [8].

Finally, Pesic et al. studied oxidative enzymes and report a multienzymatic cascade for the in situ generation of hydrogen peroxide in which a formate dehydrogenase, and an ene reductase from the old yellow enzyme family produce $\mathrm{H}_{2} \mathrm{O}_{2}$, which then serves as a reagent for the final step catalysed by a peroxygenase yielding chiral $(R)-1$-phenylethanol [9].

I very much hope that this issue meets the interest of the readers and motivates many more scientists to not only use biocatalysts, but to combine them in cascade reactions for the reasons stated above.

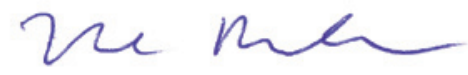

Greifswald, February 2019 Uwe Bornscheuer

\section{References}

1. Muschiol J, Peters C, Oberleitner N, Mihovilovic MD, Bornscheuer UB, Rudroff F. Cascade catalysis - strategies and challenges en route to preparative synthetic biology. Chem Comm 2015;51:5798-811.

2. Oberleitner N, Peters C, Muschiol J, Kadow M, Saß S, Bayer T, et al. An enzymatic toolbox for cascade reactions: a showcase for an in vivo redox sequence in asymmetric synthesis. ChemCatChem 2013;5:3524-8.

3. Rudroff F, Mihovilovic MD, Gröger H, Snajdrova R, Iding H, Bornscheuer UB. Opportunities and challenges for combining chemo- and biocatalysis. Nature Catal 2018;1:12-22.

4. Peters C, Buller R. Linear enzyme cascade for the production of (-)-iso-isopulegol. Z Naturforsch C 2019;74:63-70. 
5. Srinivasamurthy VS, Böttcher D, Bornscheuer UT. A multi-enzyme cascade reaction for the production of 6-hydroxyhexanoic acid.

Z Naturforsch 2019;74:71-6.

6. Ruales-Salcedo AV, Higuita JC, Fontalvo J, Woodley JM. Design of enzymatic cascade processes for the production of low-priced chemicals. Z Naturforsch 2019;74:77-84.

7. Muschiol J, Meyer AS. A chemo-enzymatic approach for the synthesis of human milk oligosaccharide backbone structures. Z Naturforsch 2019;74:85-9.
8. Farhat W, Stamm A, Robert-Monpate M, Biundo A, Syrén PO. Biocatalysis for terpene-based polymers. Z Naturforsch 2019;74:91-100.

9. Pesic M, Willot SJ, Fernández-Fueyo E, Tieves F, Alcalde $M$, Hollmann F. Multienzymatic in situ hydrogen peroxide generation cascade for peroxygenase-catalysed oxyfunctionalisation reactions. Z Naturforsch 2019;74:101-4. 